\title{
SIMPLIFYING COEFFICIENTS IN DIFFERENTIAL EQUATIONS ASSOCIATED WITH HIGHER ORDER BERNOULLI NUMBERS OF THE SECOND KIND
}

\author{
FENG QI
}

Institute of Mathematics, Henan Polytechnic University, Jiaozuo City, Henan Province, 454010, China; College of Mathematics, Inner Mongolia University for Nationalities, Tongliao City, Inner Mongolia Autonomous Region, 028043, China; Department of Mathematics, College of Science, Tianjin Polytechnic University, Tianjin City, 300387, China

\section{DA-WEI NIU}

Department of Mathematics, East China Normal University, Shanghai City, 200241, China

\section{BAI-NI GUO}

School of Mathematics and Informatics, Henan Polytechnic University, Jiaozuo City, Henan Province, 454010, China

\begin{abstract}
In the paper, by virtue of the Faà di Bruno formula, some properties of the Bell polynomials of the second kind, and an inversion formula for the Stirling numbers of the first and second kinds, the authors establish meaningfully and significantly two identities which simplify coefficients in a family of ordinary differential equations associated with higher order Bernoulli numbers of the second kind.
\end{abstract}

\footnotetext{
E-mail addresses: qifeng618@gmail.com, qifeng618@hotmail.com, qifeng618@qq.com, nnddww@gmail.com, bai.ni.guo@gmail.com, bai.ni.guo@hotmail.com

2010 Mathematics Subject Classification. Primary 34A05; Secondary 11A25, 11B68, 11B73, $11 \mathrm{~B} 83$.

Key words and phrases. simplification; coefficient; ordinary differential equation; higher order Bernoulli number of the second kind; Stirling number of the first kind; Stirling number of the second kind; inversion formula; Bell polynomial of the second kind; Faà di Bruno formula. This paper was typeset using $\mathcal{A M}_{\mathcal{S}}$-LATEX.
} 


\section{Motivations}

In [2, Theorem 1], it was inductively and recursively established that the family of differential equations

$$
(-1)^{n}(r)_{n} F(t)=[\ln (1+t)]^{n} \sum_{i=1}^{n} a_{i}(n)(1+t)^{i} F^{(i)}(t), \quad n \in \mathbb{N}
$$

has a solution

$$
F(t)=F(t, r)=\left[\frac{1}{\ln (1+t)}\right]^{r}, \quad r \in \mathbb{N},
$$

where $a_{1}(n)=1$ and

$$
a_{i}(n)=\sum_{k_{i-1}=0}^{n-i} \sum_{k_{i-2}=0}^{n-i-k_{i-1}} \cdots \sum_{k_{1}=0}^{n-i-k_{i-1}-\cdots-k_{2}} \prod_{\ell=2}^{i} \ell^{k_{\ell-1}}, \quad 2 \leq i \leq n .
$$

Let

$$
(x)_{n}=\prod_{\ell=0}^{n-1}(x+\ell)= \begin{cases}x(x+1)(x+2) \cdots(x+n-1), & n \geq 1 \\ 1, & n=0\end{cases}
$$

and

$$
\langle x\rangle_{n}=\prod_{\ell=0}^{n-1}(x-\ell)= \begin{cases}x(x-1)(x-2) \cdots(x-n+1), & n \geq 1 \\ 1, & n=0\end{cases}
$$

be the rising and falling factorials of $x \in \mathbb{R}$ for $n \in\{0\} \cup \mathbb{N}$. Let $b_{n}^{(r)}$ for $r \in \mathbb{N}$, which can be generated by

$$
\left[\frac{t}{\ln (1+t)}\right]^{r}=\sum_{n=0}^{\infty} b_{n}^{(r)} \frac{t^{n}}{n !}
$$

stand for the Bernoulli numbers of the second kind with order $r$. Theorem 2 in 2 ] reads that, if $n=0,1,2, \ldots$ and $N=1,2,3, \ldots$, then

(1) for $0 \leq n<N+r$,

$$
\begin{aligned}
(-1)^{N}(r)_{N} b_{n}^{(r+N)} & =\sum_{i=0}^{\min \{N-1, n\}} \sum_{\ell=\max \{i, n-r+1\}}^{n}\left(\begin{array}{c}
N-i \\
\ell-i
\end{array}\right)\langle n-\ell-r\rangle_{N-i}\langle n\rangle_{\ell} a_{N-i}(N) b_{n-\ell}^{(r)} ;
\end{aligned}
$$

(2) for $n \geq N+r$,

$$
\begin{aligned}
(-1)^{N}(r)_{N} b_{n}^{(r+N)}=\left(\sum_{i=0}^{\min \{n, N-1\}} \sum_{\ell=\max \{i, n-r+1\}}^{n}\right. & \left.+\sum_{i=0}^{N-1} \sum_{\ell=i}^{n-N-r+i}\right)\left(\begin{array}{c}
N-i \\
\ell-i
\end{array}\right) \\
& \times\langle n-\ell-r\rangle_{N-i}\langle n\rangle_{\ell} a_{N-i}(N) b_{n-\ell}^{(r)} .
\end{aligned}
$$

It is not difficult to see that the expression (3) of the quantity $a_{i}(n)$ is too complicated to be computed by hand and computer software. Can one find a simple, meaningful, and significant expression for the quantity $a_{i}(n)$ in $(3)$ ? 


\section{Lemmas}

For answering the above question and proving our main results, we need the following lemmas.

Lemma 1 ([1, p. 134, Theorem A] and [1, p. 139, Theorem C]). For $n \geq k \geq 0$, the Bell polynomials of the second kind, or say, partial Bell polynomials, denoted by $\mathrm{B}_{n, k}\left(x_{1}, x_{2}, \ldots, x_{n-k+1}\right)$, are defined by

$$
\mathrm{B}_{n, k}\left(x_{1}, x_{2}, \ldots, x_{n-k+1}\right)=\sum_{\substack{\ell_{1}, \ell_{2}, \ldots, \ell_{n} \in\{0\} \cup \mathbb{N} \\ \sum_{i=1}^{n} \ell_{i}=n \\ \sum_{i=1}^{n} \ell_{i}=k}} \frac{n !}{\prod_{i=1}^{n-k+1} \ell_{i} !} \prod_{i=1}^{n-k+1}\left(\frac{x_{i}}{i !}\right)^{\ell_{i}} .
$$

The Faà di Bruno formula can be described in terms of the Bell polynomials of the second kind $\mathrm{B}_{n, k}\left(x_{1}, x_{2}, \ldots, x_{n-k+1}\right)$ by

$$
\frac{\mathrm{d}^{n}}{\mathrm{~d} t^{n}} f \circ h(t)=\sum_{k=0}^{n} f^{(k)}(h(t)) \mathrm{B}_{n, k}\left(h^{\prime}(t), h^{\prime \prime}(t), \ldots, h^{(n-k+1)}(t)\right) .
$$

Lemma 2 ([1, p. 135]). For $n \geq k \geq 0$, we have

$$
\mathrm{B}_{n, k}\left(a b x_{1}, a b^{2} x_{2}, \ldots, a b^{n-k+1} x_{n-k+1}\right)=a^{k} b^{n} \mathrm{~B}_{n, k}\left(x_{1}, x_{2}, \ldots, x_{n-k+1}\right)
$$

and

$$
\mathrm{B}_{n, k}(0 !, 1 !, 2 !, \ldots,(n-k) !)=(-1)^{n-k} s(n, k),
$$

where $a$ and $b$ are any complex numbers and $s(n, k)$ for $n \geq k \geq 0$, which can be generated by

$$
\frac{[\ln (1+x)]^{k}}{k !}=\sum_{n=k}^{\infty} s(n, k) \frac{x^{n}}{n !}, \quad|x|<1,
$$

stand for the Stirling numbers of the first kind.

Lemma 3 ([21, p. 171, Theorem 12.1]). If $b_{\alpha}$ and $a_{k}$ are a collection of constants independent of $n$, then

$$
a_{n}=\sum_{\alpha=0}^{n} S(n, \alpha) b_{\alpha} \quad \text { if and only if } \quad b_{n}=\sum_{k=0}^{n} s(n, k) a_{k},
$$

where $S(n, k)$ for $n \geq k \geq 0$, which can be generated by

$$
\frac{\left(e^{x}-1\right)^{k}}{k !}=\sum_{n=k}^{\infty} S(n, k) \frac{x^{n}}{n !}
$$

stand for the Stirling numbers of the second kind.

\section{MAIN RESUlts AND THEIR PROOFS}

Now we are in a position to answer the above question and to state and prove our main results.

Theorem 1. For $n \geq 0$ and $r \in \mathbb{R}$, the function $F(t)=F(t, r)$ defined by (2) satisfies

$$
F^{(n)}(t)=\left(\frac{1}{1+t}\right)^{n}\left[\sum_{k=0}^{n} s(n, k) \frac{\langle-r\rangle_{k}}{[\ln (1+t)]^{k}}\right] F(t)
$$


and

$$
\sum_{k=0}^{n} S(n, k)(1+t)^{k} F^{(k)}(t)=\frac{\langle-r\rangle_{n}}{[\ln (1+t)]^{n}} F(t) .
$$

Proof. Let $u=u(t)=\ln (1+t)$ and $r \in \mathbb{R}$. Then, by virtue of the Faà di Bruno formula (4) and the identities (5) and (6) in sequence,

$$
\begin{aligned}
F^{(n)}(t) & =\sum_{k=0}^{n}\left(u^{-r}\right)^{(k)} \mathrm{B}_{n, k}\left(\frac{0 !}{1+t},-\frac{1 !}{(1+t)^{2}}, \ldots,(-1)^{n-k} \frac{(n-k) !}{(1+t)^{n-k+1}}\right) \\
& =\sum_{k=0}^{n} \frac{\langle-r\rangle_{k}}{u^{r+k}}\left(\frac{1}{1+t}\right)^{n}(-1)^{n+k} \mathrm{~B}_{n, k}(0 !, 1 !, \ldots,(n-k) !) \\
& =\sum_{k=0}^{n} \frac{\langle-r\rangle_{k}}{[\ln (1+t)]^{r+k}}\left(\frac{1}{1+t}\right)^{n}(-1)^{n+k}(-1)^{n-k} s(n, k) \\
& =\left(\frac{1}{1+t}\right)^{n} \frac{1}{[\ln (1+t)]^{r}} \sum_{k=0}^{n} \frac{\langle-r\rangle_{k}}{[\ln (1+t)]^{k}} s(n, k)
\end{aligned}
$$

for $n \geq 0$. Thus, the identity (7) is proved.

Applying Lemma 3 to $(7)$ leads to

$$
\frac{\langle-r\rangle_{n}}{[\ln (1+t)]^{n}} F(t)=\sum_{k=0}^{n} S(n, k)(1+t)^{k} F^{(k)}(t)
$$

which can be rewritten as 8 . The required proof is complete.

\section{REMARKS}

In this section, we give several remarks and some explanation about our main results.

Remark 1. Theorem 1 extends the range of $r$ from $\mathbb{N}$ to $\mathbb{R}$.

Remark 2. Comparing (1) with (8) reveals that

$$
a_{i}(n)=S(n, i), \quad n \geq i \geq 0 .
$$

This implies that the identity $(8)$ is more meaningful, more significant, more computable than the one (1).

Remark 3. By virtue of the expression (9), all the above mentioned results in the paper 2] can be reformulated simpler, more meaningfully, and more significantly. For the sake of saving the space and shortening the length of this paper, we do not rewrite them in details here.

Remark 4. Currently we can see that the method used in this paper is simpler, shorter, nicer, more meaningful, and more significant than the inductive and recursive method used in [2] and closely related references therein.

Remark 5. In the papers [5, 6, 18, 19, there are some new results about the Bernoulli numbers of the second kind.

Remark 6. In the papers and preprints [3, 4, 17, 8, 9, 10, 11, 12, 13, 14, 15, 16, 17, 20, 22], there are similar ideas, methods, techniques, and purposes to this paper. 
Acknowledgements. The authors thank Professor Dmitry V. Dolgy at the Far Eastern State University in Russia for his supplying an electronic version of the paper [2] through e-mail on 3 August 2017.

\section{REFERENCES}

[1] L. Comtet, Advanced Combinatorics: The Art of Finite and Infinite Expansions, Revised and Enlarged Edition, D. Reidel Publishing Co., Dordrecht and Boston, 1974.

[2] D. V. Dolgy, L.-C. Jang, D.-S. Kim, T.-K. Kim, and J.-J. Seo, Differential equations associated with higher-order Bernoulli numbers of the second kind revisited, J. Anal. Appl. 14 (2016), no. 2, 107-121.

[3] B.-N. Guo and F. Qi, Explicit formulae for computing Euler polynomials in terms of Stirling numbers of the second kind, J. Comput. Appl. Math. 272 (2014), 251-257; Available online at http://dx.doi.org/10.1016/j.cam.2014.05.018

[4] B.-N. Guo and F. Qi, Some identities and an explicit formula for Bernoulli and Stirling numbers, J. Comput. Appl. Math. 255 (2014), 568-579; Available online at http://dx.doi. org $/ 10.1016 / \mathrm{j}$.cam.2013.06.020

[5] F. Qi, A new formula for the Bernoulli numbers of the second kind in terms of the Stirling numbers of the first kind, Publ. Inst. Math. (Beograd) (N.S.) 100 (114) (2016), 243-249; Available online at http://dx.doi.org/10.2298/PIM150501028Q

[6] F. Qi, Explicit formulas for computing Bernoulli numbers of the second kind and Stirling numbers of the first kind, Filomat 28 (2014), no. 2, 319-327; Available online at http: //dx.doi.org/10.2298/FIL14023190

[7] F. Qi, Explicit formulas for the convolved Fibonacci numbers, ResearchGate Working Paper (2016), available online at http://dx.doi.org/10.13140/RG.2.2.36768.17927

[8] F. Qi and B.-N. Guo, Explicit formulas and recurrence relations for higher order Eulerian polynomials, Indag. Math. 28 (2017), no. 4, 884-891; Available online at https://doi.org/ 10.1016/j.indag.2017.06.010.

[9] F. Qi and B.-N. Guo, Some properties of a solution to a family of inhomogeneous linear ordinary differential equations, Preprints 2016, 2016110146, 11 pages; Available online at http://dx.doi.org/10.20944/preprints201611.0146.v1

[10] F. Qi and B.-N. Guo, Some properties of the Hermite polynomials and their squares and generating functions, Preprints 2016, 2016110145, 14 pages; Available online at http://dx. doi.org/10.20944/preprints201611.0145.v1

[11] F. Qi and B.-N. Guo, Viewing some ordinary differential equations from the angle of derivative polynomials, Preprints 2016, 2016100043, 12 pages; Available online at http: //dx.doi.org/10.20944/preprints201610.0043.v1

[12] F. Qi, D. Lim, and B.-N. Guo, Some identities relating to Eulerian polynomials and involving Stirling numbers, Preprints 2017, 2017080004, 10 pages; Available online at http://dx.doi. org/10.20944/preprints201708.0004.v1

[13] F. Qi, D. Lim, and B.-N. Guo, Explicit formulas and identities for the Bell polynomials and a sequence of polynomials applied to differential equations, Rev. R. Acad. Cienc. Exactas Fís. Nat. Ser. A Mat. RACSAM (2018), in press.

[14] F. Qi, D.-W. Niu, and B.-N. Guo, Simplification of coefficients in differential equations associated with higher order Frobenius-Euler numbers, Preprints 2017, 2017080017, 7 pages; Available online at http://dx.doi.org/10.20944/preprints201708.0017.v1

[15] F. Qi, J.-L. Wang, and B.-N. Guo, Notes on a family of inhomogeneous linear ordinary differential equations, Preprints 2017, 2017040026, 5 pages; Available online at http://dx. doi.org/10.20944/preprints201704.0026.v1

[16] F. Qi, J.-L. Wang, and B.-N. Guo, Simplifying and finding nonlinear ordinary differential equations, ResearchGate Working Paper (2017), available online at http://dx.doi.org/10. 13140/RG.2.2.28855.32166

[17] F. Qi, J.-L. Wang, and B.-N. Guo, Simplifying differential equations concerning degenerate Bernoulli and Euler numbers, Trans. A. Razmadze Math. Inst. 171 (2017), no. 3, in press. ResearchGate Working Paper (2017), available online at http://dx.doi.org/10.13140/RG. 2.2.12078.10566 
[18] F. Qi and X.-J. Zhang, An integral representation, some inequalities, and complete monotonicity of the Bernoulli numbers of the second kind, Bull. Korean Math. Soc. 52 (2015), no. 3, 987-998; Available online at http://dx.doi.org/10.4134/BKMS.2015.52.3.987.

[19] F. Qi and J.-L. Zhao, Some properties of the Bernoulli numbers of the second kind and their generating function, ResearchGate Working Paper (2017), available online at http: //dx.doi.org/10.13140/RG.2.2.13058.27848

[20] F. Qi, Q. Zou, and B.-N. Guo, Identities of the Chebyshev polynomials, the inverse of a triangular matrix, and identities of the Catalan numbers, ResearchGate Working Paper (2017), available online at http://dx.doi.org/10.13140/RG.2.2.27344.71684.

[21] J. Quaintance and H. W. Gould, Combinatorial Identities for Stirling Numbers. The unpublished notes of H. W. Gould. With a foreword by George E. Andrews. World Scientific Publishing Co. Pte. Ltd., Singapore, 2016.

[22] J.-L. Zhao, J.-L. Wang, and F. Qi, Derivative polynomials of a function related to the ApostolEuler and Frobenius-Euler numbers, J. Nonlinear Sci. Appl. 10 (2017), no. 4, 1345-1349; Available online at http://dx.doi.org/10.22436/jnsa.010.04.06 